\title{
Das problemzentrierte Interview
}

\section{Andreas Witzel}

Keywords:

Erhebung,

Auswertung,

Deduktion,

Induktion,

Narration, Dialog,

Leitfadeninterview,

problemzentriertes

Interview
Zusammenfassung: Das problemzentrierte Interview $(\mathrm{PZI})$ ist ein theoriegenerierendes Verfahren, das den vermeintlichen Gegensatz zwischen Theoriegeleitetheit und Offenheit dadurch aufzuheben versucht, dass der Anwender seinen Erkenntnisgewinn als induktiv-deduktives Wechselspiel organisiert. Entsprechende Kommunikationsstrategien zielen zum einen auf die Darstellung der subjektiven Problemsicht. Zum anderen werden die angeregten Narrationen durch Dialoge ergänzt, die Resultat ideenreicher und leitfadengestützter Nachfragen sind. Theoretisches Wissen entsteht im Auswertungsprozess durch Nutzen elastischer Konzepte, die in der empirischen Analyse fortentwickelt und mit empirisch begründeten "Hypothesen" am Datenmaterial erhärtet werden.

\section{Inhaltsverzeichnis}

1. Einleitung

2. Die Grundpositionen des problemzentrierten Interviews

3. Die Instrumente des problemzentrierten Interviews

4. Die Gestaltung des problemzentrierten Interviews

$\underline{5 .}$ Auswertung des problemzentrierten Interviews

Literatur

Zum Autor

Zitation

\section{Einleitung}

Die Konstruktionsprinzipien des problemzentrierten Interview (PZI) (WITZEL 1982, 1985) ${ }^{1}$ zielen auf eine möglichst unvoreingenommene Erfassung individueller Handlungen sowie subjektiver Wahrnehmungen und Verarbeitungsweisen gesellschaftlicher Realität. [1]

Empirische Untersuchungen aus der Akteursperspektive und damit qualitative Interviews bekommen durch die "Individualisierungsthese" (u.a. BECK 1986) eine neue Bedeutsamkeit. Dieser These zufolge lösen sich Individuen aus alten Bindungen (Stände, Klassen etc.) und müssen sich neuen institutionellen Abhängigkeiten wie Arbeitsmarkt, Beruf oder Bildung stellen. Die Möglichkeit, Handlungsresultate unmittelbar aus gesellschaftlichen Schranken,

Selektionsmechanismen und sozial ungleich verteilten Ressourcen zu erklären, schwindet. Die Akteure müssen vielmehr die Folgen ihres Handelns selbst verantworten. Wenn sie diese Zuschreibung für sich akzeptieren und sich zunehmend als "Planungsbüro" ihres Lebenslauf betrachten, ist Selbstreflexion in gewissem Umfang notwendig. Auch neuere Konzepte der Sozialisationsforschung wie das der "Selbstsozialisation" (HEINZ \& WITZEL

1 Empfehlenswerte Zusammenfassungen: MEY (1999, S.142-150), LAMNEK (1989, S.74-78). Hinweise zur Durchführung der Erhebung und der Auswertung am praktischen Beispiel: SCHMIDT-GRUNERT (1999). 
1995) verweisen gegenüber sozialnormativen Ansätzen auf Eigenleistungen des Subjekts im Umgang mit gesellschaftlichen Gegebenheiten, beim Entwickeln eigener Ansprüche in Beruf, Partnerschaft und Familie, bei der Verarbeitung von biographischen Erfahrungen sowie beim Nutzen von eigenen Ressourcen und Handlungsspielräumen für private und berufliche Pläne. [2]

\section{Die Grundpositionen des problemzentrierten Interviews}

Das PZI lehnt sich weitgehend an das theoriegenerierende Verfahren der "Grounded Theory" (GLASER \& STRAUSS 1998) an, das zum einen die Kritik an einer hypothetico-deduktiven Vorgehensweise einlöst, derzufolge man die Daten nur durch ex ante festgelegte Operationalisierungsschritte erfassen und überprüfen kann. Zum anderen wendet es sich aber auch gegen die naivinduktivistische Position des "soziologischen Naturalismus" (z.B. HOFFMANNRIEM 1980), derzufolge die Haltung des Interviewers/Wissenschaftlers durch prinzipielle Offenheit gegenüber der Empirie gekennzeichnet ist bzw. unter Ausklammerung des theoretischen Vorwissens als tabula rasa konzeptioniert wird (vgl. KELLE 1996). Bezogen auf das PZI ist der Erkenntnisgewinn sowohl im Erhebungs- als auch im Auswertungsprozess vielmehr als induktiv-deduktives Wechselverhältnis zu organisieren. Das unvermeidbare, und damit offenzulegende Vorwissen dient in der Erhebungsphase als heuristisch-analytischer Rahmen für Frageideen im Dialog zwischen Interviewern und Befragten. Gleichzeitig wird das Offenheitsprinzip realisiert, indem die spezifischen Relevanzsetzungen der untersuchten Subjekte insbesondere durch Narrationen angeregt werden. Theoretisches Wissens entsteht in der Auswertungsphase durch Nutzen von "sensitizing concepts" (BLUMER 1954, S.7), die in der weiteren Analyse fortentwickelt und mit empirisch begründeten Hypothesen am Datenmaterial erhärtet werden. Mit dieser elastischen Vorgehensweise soll gewährleistet werden, dass die Problemsicht des Interviewers/Wissenschaftlers nicht diejenige der Befragten überdeckt, und den erhobenen Daten nicht im Nachhinein einfach Theorien "übergestülpt" werden. [3]

Vor diesem Hintergrund lassen sich die drei Grundpositionen des PZI wie folgt skizzieren:

- Die Problemzentrierung kennzeichnet die Orientierung an einer gesellschaftlich relevanten Problemstellung und charakterisiert die Organisation des Erkenntnis- oder Lernprozesses (Vorinterpretation): Der Interviewer nutzt die vorgängige Kenntnisnahme von objektiven Rahmenbedingungen ${ }^{2}$ der untersuchten Orientierungen und Handlungen, um die Explikationen der Interviewten verstehend nachzuvollziehen und am Problem orientierte Fragen bzw. Nachfragen zu stellen. Parallel zur Produktion von breitem und differenziertem Datenmaterial arbeitet der Interviewer schon an der Interpretation der subjektiven Sichtweise der

2 Handlungskontexte wie Schul-, Berufs- und Arbeitsmarktorganisation, Geschlecht, Schicht oder regionale Besonderheiten sind objektiv insoweit, als sie dem individuellen Handeln vorausgesetzt und von den Akteuren nicht veränderbar sind. 
befragten Individuen und spitzt die Kommunikation immer präziser auf das Forschungsproblem zu.

- Die Gegenstandsorientierung betont die Flexibilität der Methode gegenüber den unterschiedlichen Anforderungen des untersuchten Gegenstands. Daher wurde das PZI in den Zusammenhang einer Methodenkombination gestellt, innerhalb derer das Interview das wichtigste Instrument bildet. So kann es etwa sinnvoll sein, sich auf Interviews mit einer neuen Forschungsthematik dahingehend vorzubereiten, dass man zunächst eine Gruppendiskussion durchführt, um einen ersten Überblick über Meinungsinhalte in der zu untersuchenden Stichprobe zu bekommen. Die biographische Methode verweist etwa auf den Aspekt der Entwicklung von Deutungsmustern im Rahmen der individuellen Auseinandersetzung mit sozialer Realität. Zuletzt lässt sich das Interview auch mit einem standardisierten Fragebogen zur Lösung von Stichprobenproblemen (als Beispiel: MÖNNICH \& WITZEL 1994) und zur Kombination der mit den unterschiedlichen Verfahren gewonnenen Ergebnisse verbinden (als Beispiel ERZBERGER 1998, S.153ff). Auch die Gesprächstechniken werden flexibel eingesetzt: Den Erfordernissen des Aufbaus einer befragtenzentrierten Kommunikationssituation folgend kann der Interviewer je nach der unterschiedlich ausgeprägten Reflexivität und Eloquenz der Befragten stärker auf Narrationen oder unterstützend auf Nachfragen im Dialogverfahren setzen.

- Die Prozessorientierung bezieht sich auf den gesamten Forschungsablauf und insbesondere auf die Vorinterpretation. Wenn der

Kommunikationsprozess sensibel und akzeptierend auf die Rekonstruktion von Orientierungen und Handlungen zentriert wird, entsteht bei den Befragten Vertrauen und damit Offenheit, weil sie sich in ihrer Problemsicht ernst genommen fühlen. Dieses Vertrauensverhältnis fördert die Erinnerungsfähigkeit und motiviert zur Selbstreflexion. Indem die Befragten ihre Problemsicht "ungeschützt" in Kooperation mit dem Interviewer entfalten, entwickeln sie im Laufe des Gesprächs immer wieder neue Aspekte zum gleichen Thema, Korrekturen an vorangegangenen Aussagen, Redundanzen, und Widersprüchlichkeiten. Redundanzen sind insofern erwünscht, als sie oft interpretationserleichternde Neuformulierungen enthalten.

Widersprüchlichkeiten drücken individuelle Ambivalenzen und Unentschiedenheiten aus, die thematisiert werden sollten. Ihnen liegen möglicherweise Missverständnisse des Interviewers oder Fehler und Lücken in der Erinnerung der Interviewten zugrunde, die durch Nachfragen aufgeklärt werden können. Sie können aber auch Ausdruck von Orientierungsproblemen, Interessenswidersprüchen und Entscheidungsdilemmata angesichts widersprüchlicher Handlungsanforderungen sein. Die Förderung der Gesprächsentwicklung wird gerade durch die Anwendung des PZI als biographisches Interview deutlich, wenn Erzählungen von Lebensgeschichten oder erlebten Begebenheiten angeregt werden. Als eine ursprüngliche Form der Reflexion bauen Erzählungen die Künstlichkeit der Forschungssituation ab und verhindern, dass die Befragten gezwungen sind, "isolierte Antworten auf isolierte Fragen" (BAHRDT 1975, S.13, vgl. auch BERGER 1974) geben zu müssen. Dieses 
z.B. in Befragungen der Meinungsforschung übliche Frage-Antwort-Spiel institutionalisiert geradezu den Verzicht auf eine systematische Entwicklung des Problemhorizonts der Befragten (CICOUREL 1974, S.97). [4]

\section{Die Instrumente des problemzentrierten Interviews}

Vier Instrumente ermöglichen und unterstützen die Durchführung des PZI: Kurzfragebogen, Leitfaden, Tonaufzeichnung des Gesprächs und Postskriptum. [5]

Der Kurzfragebogen dient zum einen der Ermittlung von Sozialdaten (Alter, Beruf der Eltern usw.). Das nachfolgende Interview, das eine Aushandlung der subjektiven Sichtweise der Interviewten zum Ziel hat, wird von denjenigen Fragen entlastet, die als Frage-Antwort-Schema aufgebaut sind. Zum anderen können die in inm enthaltenen Informationen - und insbesondere in Kombination mit einer offenen Frage - einen Gesprächseinstieg ermöglichen. So lassen sich etwa Berufswunschangaben für eine Einleitungsfrage zum untersuchten Problemfeld des Übergangs Jugendlicher von der Schule in die Ausbildung nutzen (zu beiden Aspekten siehe die folgenden Ausführungen in Abschnitt 4). [6]

Die im allgemeinen von den Interviewten akzeptierte Tonträgeraufzeichnung erlaubt im Gegensatz etwa zu Gesprächsprotokollen die authentische und präzise Erfassung des Kommunikationsprozesses; sie sollte anschließend vollständig transkribiert werden. Der Interviewer kann sich ganz auf das Gespräch sowie auf Beobachtungen situativer Bedingungen und nonverbaler Äußerungen konzentrieren. [7]

Im Leitfaden sind die Forschungsthemen als Gedächtnisstütze und Orientierungsrahmen zur Sicherung der Vergleichbarkeit der Interviews festgehalten. Darüber hinaus sind einige Frageideen zur Einleitung einzelner Themenbereiche und eine vorformulierte Frage zum Gesprächsbeginn enthalten. Im Idealfall begleitet der Leitfaden den Kommunikationsprozess als eine Art Hintergrundfolie, die zur Kontrolle dient, inwieweit seine einzelnen Elemente im Laufe des Gesprächs behandelt worden sind. [8]

Als Ergänzung zur Tonträgeraufzeichnung werden unmittelbar nach dem Gespräch Postskripte (vgl. auch CICOUREL 1974) erstellt. Sie enthalten eine Skizze zu den Gesprächsinhalten, Anmerkungen zu den o.g. situativen und nonverbalen Aspekten sowie zu Schwerpunktsetzungen des Interviewpartners. Außerdem werden spontane thematische Auffälligkeiten und Interpretationsideen notiert, die Anregungen für die Auswertung geben können. Postskripte werden auch genutzt, um in Anlehnung an das Verfahren der "theoretischen Stichprobe" (GLASER \& STRAUSS 1998) Kriterien für eine inhaltlich begründete Auswahl von Einzelfallanalysen zu entwickeln. Damit können sukzessive Kontrastfälle gebildet, sowie Ähnlichkeiten und Gegenevidenzen gesucht werden. [9] 


\section{Die Gestaltung des problemzentrierten Interviews}

Zunächst ist die unmittelbare Kontaktaufnahme Teil des Interviewablaufs. Die weitere Gestaltung des Gesprächs erfolgt dann zum einen mit den erzählungsgenerierenden Kommunikationsstrategien Gesprächseinstieg, allgemeine Sondierungen und Ad-hoc-Fragen; zum anderen mit den verständnisgenerierenden Strategien der spezifischen Sondierungen mit den Elementen Zurückspiegelungen, Verständnisfragen und Konfrontationen. [10]

Neben der Zusicherung der Anonymisierung der Gesprächsprotokolle und der Erklärung der gewünschten Gesprächsform wird während der Kontaktaufnahme die Untersuchungsfrage erläutert. Der Interviewer versucht also, sich hypothetischen Etikettierungen des Untersuchungszwecks (vgl. BERGER 1974) zu entziehen, indem er sein Erkenntnisinteresse offen legt und deutlich macht, dass die Explikationen der Interviewten nicht als Ausdruck von intellektuellen Leistungen bewertet, sondern als individuelle Vorstellungen und Meinungen akzeptiert werden. [11]

Das PZI ist ein "diskursiv-dialogisches Verfahren" (MEY 1999, S.145), das - wie schon in der Kontaktaufnahme zum Ausdruck kommt - die Befragten als Experten ihrer Orientierungen und Handlungen begreift, die im Gespräch die Möglichkeit zunehmender Selbstvergewisserung mit allen Freiheiten der Korrektur eigener oder der Intervieweraussagen wahrnehmen können. Um seinen eigenen Erkenntnisfortschritt zu optimieren, kombiniert der Interviewer das Zuhören mit Nachfragen. Hierzu stehen die im folgenden dargestellten Gesprächstechniken zur Verfügung, die flexibel eingesetzt werden. [12]

\section{a) Erzählungsgenerierende Kommunikationsstrategien:}

Eine vorformulierte Einleitungsfrage ist ein Mittel der Zentrierung des Gesprächs auf das zu untersuchende Problem. Zugleich soll die Frage so offen formuliert sein, dass sie für den Interviewten "wie eine leere Seite" wirkt, die er in eigenen Worten und mit den ihm eigenen Gestaltungsmitteln füllen kann. Eine solche Frage könnte - an die o.g. Frage nach dem Berufswunsch anknüpfend lauten: "Sie möchten Friseuse werden. Wie sind Sie darauf gekommen (oder: wie kam es dazu)? Erzählen Sie doch mal!" Der letzte Satz fordert noch einmal explizit zur Abkehr vom traditionellen und von den meisten Interviewten zunächst erwarteten Interviewstil des Frage-Antwort-Spiels auf. [13]

Im weiteren Verlauf der Kommunikation dienen allgemeine Sondierungen einer sukzessiven Offenlegung der subjektiven Problemsicht (Prinzip der Offenheit oder Induktion). Der Interviewer greift die thematischen Aspekte der auf die Einleitungsfrage folgenden Erzählsequenz auf, um mit entsprechenden Nachfragen den roten Faden weiterzuspinnen und zu detaillieren, den die Befragten in dieser Sequenz angeboten haben. Gleichzeitig dokumentieren diese Nachfragen den gewünschten Grad der Detaillierung der angesprochenen Thematik. Ein "Hervorlocken" konkreter Erfahrungsbeispiele oder biographischer Episoden regt die Erinnerungsfähigkeit an, verdeutlicht abstrakte, fehlende oder 
unklare Begriffe und stellt konkrete Bezüge zu Kontextbedingungen des Handelns her. [14]

Ad-hoc-Fragen werden notwendig, wenn bestimmte Themenbereiche, die auch die Vergleichbarkeit der Interviews sichern, von den Interviewten ausgeklammert wurden. Sie ergeben sich aus Stichworten im Leitfaden oder können auch einzelne standardisierte Fragen beinhalten, die zur Vermeidung des FrageAntwort-Spiels im Hauptteil des Interviews am Ende des Gesprächs gestellt werden, so beispielsweise die Frage: "Was möchten Sie erreicht haben, wenn Sie mal 30 Jahre alt geworden sind?" [15]

\section{b) Verständnisgenerierende Kommunikationsstrategien:}

Bei den spezifischen Sondierungen macht sich der Aspekt der Deduktion geltend, indem der Interviewer das vorgängige oder im Interview selbst erworbene Wissen für Frageideen nutzt: Die an die Gesprächspsychotherapie angelehnte Zurückspiegelung von Äußerungen der Befragten stützt deren Selbstreflexion und eröffnet innen die Möglichkeit, ihre eigene Sichtweise zu behaupten und die Unterstellungen des Interviewers zu korrigieren (kommunikative Validierung). Klärende Verständnisfragen werden bei ausweichenden oder widersprüchlichen Antworten angewandt und brechen Alltagsselbstverständlichkeiten der Interviewten auf. Konfrontationen können weitere Detaillierungen von Sichtweisen der Befragten fördern. Allerdings muss ein gutes Vertrauensverhältnis hergestellt worden sein, um keine Rechtfertigungen zu provozieren. [16]

Die Frage, wann es sinnvoll ist, von den erzählungsgenerierenden zu den verständnisgenerierenden Fragen zu wechseln, lässt sich prinzipiell an der aus der Ethnomethodologie stammenden "dokumentarischen Methode der Interpretation" (GARFINKEL 1962) verdeutlichen. Die Methode kennzeichnet einen hermeneutischen Prozess, der im Alltag die Wirklichkeitskonzeptionen der Individuen konstituiert und ihre gemeinsame Handlungspraxis ermöglicht. Sie wurde auf das sozialwissenschaftliche Anliegen, Sinnstrukturen zu ermitteln, übertragen: Die Zuordnung von Einzelaspekten der Erzählungen zu vorgängigen Mustern der Sinninterpretation, die der Interviewer in das Gespräch einbringt (Deduktion), wird ergänzt durch die Suche nach neuen Mustern für die mit diesen vorgängigen Mustern nicht zu erklärenden Einzelphänomene in den Darlegungen des Interviewten (Induktion). Konkret fördert der Interviewer Narrationen durch erzählungsgenerierende Fragen und wartet dabei ab, bis einzelne Äußerungen sich zu einem Muster fügen. Umgekehrt können mit den unterschiedlichen verständnisgenerierenden Fragetechniken neue Muster des Sinnverstehens entstehen oder alte Muster durch spätere Detailäußerungen oder Kontrollen des Interviewten korrigiert werden. Diese komplexe Gesprächsstrategie (das Vorwissen für Fragen zu nutzen, ohne damit die originäre Sichtweise der Befragten zu überdecken) stellt hohe Anforderungen an den Interviewer. Der Wissenschaftler sollte deshalb die Interviews möglichst selbst durchführen und sie nicht an Hilfskräfte oder Umfrage-Institute weitergeben. [17] 


\section{Auswertung des problemzentrierten Interviews}

Dem Prinzip der Gegenstandsorientierung entsprechend gibt es für unterschiedliche Erkenntnisinteressen und thematischen Bezüge verschiedene Auswertungsmethoden. Der folgende Vorschlag, der sorgfältige Einzelfallanalysen mit einer Typenbildung auf der Basis relativ großer Fallzahlen zu verbinden sucht, bezieht sich auf langjährige eigene Erfahrungen in Forschungsprojekten mit sozialisations- und lebenslauftheoretischen Fragestellungen (z.B. HEINZ et al. 1987, KÜHN \& WITZEL 1999). [18]

Grundlage aller Auswertungsarbeit (WITZEL 1996) ist die Fallanalyse auf der Basis vollständig transkribierter Interviews. Dabei bezieht sich der erste Schritt konsequenterweise auf die bereits im Verlauf der Erhebung initiierten Vorinterpretationen, die der Auswerter Satz für Satz deutend nachvollzieht. Die Resultate dieses Auswertungsprozesses bestehen zunächst in der Markierung des Textes mit Stichworten aus dem Leitfaden (theoriegeleitet) und mit Begrifflichkeiten, die neue thematische Aspekte aus den Darstellungen der Interviewpartner kennzeichnen (induktiv). [19]

Diese Markierungen können auch Grundlage der Entwicklung eines Codierrasters für den Aufbau einer Textdatenbank sein, die als elektronisches Fundstellenregister mit komplexen Zugriffsmöglichkeiten genutzt werden kann. Mithilfe von "Retrievals", d.h. über eine Datenbank hergestellte Verknüpfungen von Schlagwörtern ("Codes") oder Variablen (wie Geschlecht, Beruf) mit Textpassagen (vgl. PREIN 1996), lassen sich dann unter verschiedenartigsten Aspekten Originaltextstellen finden oder Querverbindungen zwischen unterschiedlichen Textstellen und Einzelfällen herstellen (KÜHN \& WITZEL 1999). [20]

Des weiteren erfolgt eine analytische Zuordnung thematischer Auffälligkeiten zu "In-vivo-codes", d.h. alltagsnahen Begriffen. Diese Auswertungsideen können Eingang finden in Notizen oder kleinen Ausarbeitungen, die GLASER und STRAUSS (1998) "Memos" nennen. [21]

Der nächste Schritt der Fallanalyse besteht im Verfertigen einer Falldarstellung oder biographischen Chronologie, die den Interpreten mit dem Einzelfall vertraut machen soll. Mit innen lassen sich in der weiteren Analyse Einzelaussagen oder Textsequenzen in einen Gesamtzusammenhang, z.B. eines biographischen Verlaufs stellen. Diese Gesamtgestalt erleichtert auch den Einstieg in die ständig notwendigen Re-Analysen. [22]

Das Dossier bzw. die Fallbewertung enthält einen Kommentar des Auswerters über die Beschaffenheit des vorliegenden Interviewmaterials, die Besonderheiten des Falls, interpretative Unsicherheiten, außergewöhnliche Ereignisabläufe und methodische Fehler. [23]

Fallspezifische zentrale Themen stellen erste Ergebnisse des theoriegenerierenden Interpretationsschritts mit themen- oder biographieorientierten Auswertungsideen dar. Sie werden zu einer prägnanten Aussage verdichtet und 
verbinden Originaltextstellen, Paraphrasierungen und analytische Aussagen ("offene Kodierung", STRAUSS \& CORBIN 1990). Gleichzeitig können Heuristiken wie z.B. ein handlungstheoretisches Modell für die themenspezifische Nachvollziehbarkeit der subjektiven Logik des Falls nützlich sein ("axiale Kodierung", dies.). Dies zeigt wiederum, wie eine offene und eine theoriegeleitete Vorgehensweise miteinander verschränkt sind. Diese Stufe der theoretischen Begriffsbildung wird anschließend individuell am Text und dann im Auswertungsteam diskursiv validiert. [24]

Der systematisch kontrastierende Fallvergleich zielt zunächst auf die Erarbeitung fallübergreifender zentraler Themen. Dabei werden die Einzelfälle in ihren inhaltlichen Ausprägungen und Merkmalen wie Geschlecht, Region und Beruf nach dem Prinzip "maximaler und minimaler Kontrastierung" (GERHARD 1986, S.69) miteinander verglichen und Ähnlichkeiten und Gegenevidenzen gesucht. Interessante Problembereiche, Querverbindungen etc. werden herausgearbeitet und in Memos festgehalten. Ziel ist dabei, "Kernkategorien" (STRAUSS \& CORBIN 1990) z.B. in Form eines Typologiekonzeptes zu entwickeln, die dann in der nächsten Auswertungsstufe des "selektiven Kodierens" (vgl. dies.) als Deutungshypothese genutzt, nunmehr theoriegeleitet oder deduktiv mit weiterem empirischen Material aufgefüllt werden. [25]

\section{Literatur}

Bahrdt, Hans P. (1975). Erzählte Lebensgeschichten von Arbeitern. In Martin Osterland (Hrsg.), Arbeitssituation, Lebenslage und Konfliktpotential. Frankfurt a.M.: EVA.

Beck, Ulrich (1986). Risikogesellschaft. Auf dem Weg in eine andere Moderne. Frankfurt a.M.: Suhrkamp.

Berger, Hartwig (1974). Untersuchungsmethode und soziale Wirklichkeit. Frankfurt a.M.: Suhrkamp. Blumer, Herbert (1954). What is wrong with social research. American Sociological Review, 14, 3-10. Cicourel, Aron V. (1974). Theory and method in a study of Argentine fertility. New York: Wiley Erzberger, Christian (1998). Zahlen und Wörter. Die Verbindung quantitativer und qualitativer Daten und Methoden im Forschungsprozeß. Weinheim: Deutscher Studien Verlag.

Garfinkel, Harold (1962). Common sense knowledge of social structures: The documentary method of interpretation in lay and professional fact finding. In Jordan M. Scher (Hrsg.), Theories of the mind (S.689-712). New York: The Free Press of Glencoe.

Gerhardt, Uta (1986). Verstehende Strukturanalyse: Die Konstruktion von Idealtypen als Analyseschritt bei der Auswertung qualitativer Forschungsmaterialien. In Hans-Georg Soeffner (Hrsg.), Sozialstruktur und Soziale Typik (S.31-83). Frankfurt a.M.: Campus.

Glaser, Barney G. \& Strauss, Anselm G. (1998). Grounded Theory. Strategien qualitativer Forschung. Bern: Huber (Original 1967).

Heinz, Walter. R.; Krüger, Helga; Rettke, Ursula; Wachtveitl, Erich \& Witzel, Andreas (1987).

"Hauptsache eine Lehrstelle". Jugendliche vor den Hürden des Arbeitsmarktes. Weinheim: Deutscher Studien Verlag.

Heinz, Walter R. \& Witzel, Andreas (1995). Das Verantwortungsdilemma in der beruflichen Sozialisation. In Ernst.-H. Hoff \& Lothar Lappe (Hrsg.), Verantwortung im Arbeitsleben (S.99-113). Weinheim: Asanger.

Hoffmann-Riem, Christel (1980). Die Sozialforschung einer interpretativen Soziologie - Der Datengewinn. Kölner Zeitschrift für Soziologie und Sozialpsychologie, 32, 339-372.

Kelle, Udo (1996). Die Bedeutung theoretischen Vorwissens in der Methodologie der Grounded Theory. In Rainer Strobl \& Andreas Böttger (Hrsg.), Wahre Geschichten? Zur Theorie und Praxis qualitativer Interviews (S.23-48). Baden Baden: Nomos. 
Kühn, Thomas \& Witzel, Andreas (1999). Computergestützte Auswertung biographischer Interviews mittels einer qualitativen Datenbank. Erscheint in der Reihe Arbeitspapiere des Sonderforschungsbereichs 186 der Universität Bremen.

Lamnek, Siegfried (1989). Qualitative Sozialforschung. Band 2: Methoden und Techniken. München: Deutsche Verlags Union.

Mey, Günter (1999). Adoleszenz, Identität, Erzählung. Theoretische, methodische und empirische Erkundungen. Berlin: Köster.

Mönnich, Ingo \& Witzel, Andreas (1994). Arbeitsmarkt und Berufsverläufe junger Erwachsener. Ein Zwischenergebnis. Zeitschrift für Sozialisationsforschung und Erziehungssoziologie, 14, 3, 263-278.

Prein, Gerald (1996). Interpretative Methodologie und Computer. Kann EDV-gestützte Datenverwaltung Validitätsprobleme qualitativer Forschung lösen? In Rainer Strobl \& Andreas Böttger (Hrsg.), Wahre Geschichten? Zur Theorie und Praxis qualitativer Interviews (S.93-110). Baden Baden: Nomos.

Schmidt-Grunert, Marianne (Hrsg.) (1999). Sozialarbeitsforschung konkret: problemzentrierte Interviews als qualitative Erhebungsmethode. Freiburg: Lambertus.

Strauss, Anselm \& Corbin, Juliet M. (1990). Basics of qualitative research, grounded theory, procedures and techniques. Newbury Park: Sage.

Witzel, Andreas (1982). Verfahren der qualitativen Sozialforschung. Überblick und Alternativen. Frankfurt a.M.: Campus.

Witzel, Andreas (1989). Das problemzentrierte Interview. In Gerd Jüttemann (Hrsg.), Qualitative Forschung in der Psychologie. Grundfragen, Verfahrensweisen, Anwendungsfelder (S.227-256). Heidelberg: Asanger.

Witzel, Andreas (1996) Auswertung problemzentrierter Interviews. Grundlagen und Erfahrungen. In Rainer Strobl \& Andreas Böttger (Hrsg.), Wahre Geschichten? Zur Theorie und Praxis qualitativer Interviews (S.49-76). Baden Baden: Nomos.

Witzel, Andreas \& Kühn, Thomas (1999). Berufsbiographische Gestaltungsmodi. Eine Typologie der Orientierungen und Handlungen beim Übergang in das Erwerbsleben. Arbeitspapiere des Sonderforschungsbereichs 186 der Universität Bremen, Nr.61. Als PDF-Datei abrufbar unter http:// www.sfb186.uni-bremen.de/download/paper61.pdf (Broken link, FQS, May 2003)

\section{Zum Autor}

Andreas WITZEL, Dr. phil., Dipl.-Psych.; geboren 1945; Studium in Regensburg und Darmstadt; wissenschaftlicher Mitarbeiter an der Universität Bremen; seit 1990 im Sonderforschungsbereich 186 "Statuspassagen und Risikolagen im Lebensverlauf" (hierzu weitere Informationen unter: http://www.sfb186.uni-bremen.de).

Interessen: Berufliche und vorberufliche Sozialisationsforschung, insbesondere Umgangsweisen Jugendlicher und junger Erwachsener mit ihrer Berufsbiographie und Verarbeitungsweisen gesellschaftlicher Realität; Methoden der interpretativen Sozialforschung, insbesondere qualitative Interview- und Auswertungsverfahren und Verknüpfung qualitativer und quantitativer Methoden.
Kontakt:

Andeas Witzel

Sonderforschungsbereich 186

Wiener Straße, Postfach 330440

D-28334 Bremen

Tel.: +49 / (0)421 / 2184141

Fax: +49 / (0)421 / 2184153

E-Mail: awitzel@sfb186.uni-bremen.de

\section{Zitation}

Witzel, Andreas (2000). Das problemzentrierte Interview [25 Absätze]. Forum Qualitative Sozialforschung / Forum: Qualitative Social Research, 1(1), Art. 22, http://nbnresolving.de/urn:nbn:de:0114-fqs0001228. 